\title{
Flow and Transport in Porous Media: A Multiscale Focus
}

\author{
Amgad Salama, ${ }^{1}$ Shuyu Sun, ${ }^{2}$ Mohamed F. El Amin, ${ }^{3}$ Yi Wang, ${ }^{4}$ and Kundan Kumar ${ }^{5}$ \\ ${ }^{1}$ University of Regina, Regina, SK, Canada \\ ${ }^{2}$ King Abdullah University of Science and Technology, Thuwal, Jeddah, Saudi Arabia \\ ${ }^{3}$ Effat University, Jeddah, Saudi Arabia \\ ${ }^{4}$ National Engineering Laboratory for Pipeline Safety/MOE Key Laboratory of Petroleum Engineering, \\ Beijing Key Laboratory of Urban Oil and Gas Distribution Technology, University of Petroleum, Beijing 102249, China \\ ${ }^{5}$ University of Bergen, Bergen, Norway \\ Correspondence should be addressed to Amgad Salama; amgad.salama@uregina.ca
}

Received 10 September 2017; Accepted 14 September 2017; Published 26 October 2017

Copyright (C) 2017 Amgad Salama et al. This is an open access article distributed under the Creative Commons Attribution License, which permits unrestricted use, distribution, and reproduction in any medium, provided the original work is properly cited.

Porous media are ubiquitous in almost all of our daily life applications, from small scale biological cell membranes to field scale subsurface reservoirs (e.g., groundwater, petroleum, and geothermal) and beyond. It is indeed a challenge to account for such vast pool of length scales in a unified framework. In fact, the continuum hypothesis furnishes a suitable framework for the study of problems related to porous media applications. However, there exist situations of several interesting applications that occur at the boundaries between different scales (e.g., in fractured media). Phenomena that develop across the boundaries of different scales are, generally, difficult to handle. Variables at different scales have been recognized to have different meanings, interpretations, and measuring windows. Therefore, there is currently a large interest, among researchers, to develop theories and algorithms that are able to link these scales in a seamless manner. Meanwhile, the success to adapt the continuum hypothesis rely, to a large extent, on our understanding of the underlying physics at the small scale. As an example, the attempts to generalize the use of traditional Darcy's law on problems related to transport phenomena in tight formations (e.g., shales) necessitated the need to revisit the physics involved during the flow and transport in nanoscale passages such that correct upscaling to field applications is realized. Furthermore, new emerging applications including the transport of nanomaterials, new emerging contaminants, and new remediation technologies require, probably, newer insight into the way we model porous media problems. We, therefore, solicited high quality original research or review articles focusing on all aspects of flow and transport in porous media to be submitted to this special issue. The aim has been to bring together state-of-theart research contributions on the rich topics related to transport phenomena in porous media with emphasis on length scale span. Several applications have been thought including subsurface petroleum reservoirs, groundwater flows, shale gas transport, flows in tight formations, pore scale modeling, coupled flow and heat transfer problems, and numerical algorithms. The call for contributions has found particular interest among researchers and we needed to extend the time of the special issue per the many requests we received from interested scholars. We received a total of 22 submissions, and after two rounds of rigorous review, 13 papers were accepted one of which is a review article.

In the paper "Computing and Comparing Effective Properties for Flow and Transport in Computer-Generated Porous Media," R. Allen and S. Sun conducted pore scale simulation to examine, numerically, effective properties (i.e., permeability, hydraulic tortuosity, and diffusive tortuosity) of three different digital porous media samples, including in-line array of uniform shapes, staggered-array of squares, and randomly distributed squares. They highlighted that hydraulic and diffusive tortuosity can be quantitatively different by up to a factor of ten in the same pore geometry, which indicates these tortuosity terms cannot be used interchangeably. They also find when a pore geometry is characterized by an anisotropic permeability, the diffusive tortuosity (and correspondingly the effective diffusion coefficient) can also be anisotropic. 
In the paper "A Pore-Scale Simulation on ThermalHydromechanical Coupling Mechanism of Rock," R. Song et al. presented a pore scale thermal-hydromechanical coupling study of the flow of immiscible two-phase system in a perfect-plastic rock. A rock matrix and pore space system are reconstructed using micro-CT image. The rock deformation and fluid flow are simulated using ANSYS and CFX software, respectively. They highlight that the rising of effective pressure or temperature would lead to a decline of the porosity and permeability and that the drop ratio of permeability is larger than that of porosity. They also found that the relative permeability of oil and water decreases with the increasing of the effective pressure.

In the paper "Determining the REV for Fracture Rock Mass Based on Seepage Theory," L. Zhang et al. indicated that the equivalent porous medium approximation may be the main method in the study of the seepage in fractured rock mass system. The key to the method is the ability to define a representative elementary volume (REV). They highlight that not all types of fractured rock mass have REV. The more intensive the fractures are, the better the penetration and the better the permeability of the rocks, which means the easier it is to become equivalent to porous media.

In the paper "Logging Characteristics and Identification Methods of Low Resistivity Oil Layer: Upper Cretaceous of the Third Member of Qingshankou Formation, Daqingzijing Area, Songliao Basin, China," C. Feng et al. highlight the importance of the identification and evaluation of low resistivity oil layer in logging interpretation. This is mainly due to small resistivity contrast between oil and water layers. The study focuses on low resistivity thick layer sandstone in Qingshankou Formation at Daqingzijing oilfield, along with comprehensive data of logging, core, oil test, and production test.

In the paper "Modelling of River-Groundwater Interactions under Rainfall Events Based on a Modified Tank Model," W. Nie et al. combined experiments and modelling to develop a novel tank model to simulate the ground water table and river levels due to rainfall events. The parameter training of the model used learning algorithms to predict the pore water pressure using laboratory conditions.

In the paper "Experimental Study on the Effects of Stress Variations on the Permeability of Feldspar-Quartz Sandstone," F. Wang et al. studied the impact of injection processes of $\mathrm{CO}_{2}$ sequestration on the hydraulic properties of reservoirs. The repeated loading and unloading involved in the multistage injection process implied that the hydraulic properties such as permeability evolved with time. An interesting observation from this study is the effect of multiple stress cycles on the micropore structure and the clay mineral content having a close relationship with the microscopic pore structure of the rock. This showed that the permeability of rocks of the same type with different clay mineral contents displayed different responses to stress.

In the paper "Fluid Flow and Heat Transport Computation for Power-law Scaling Poroperm Media," P. Leary et al. implemented finite-element modelling methodology for a Dm-scale fracture sequence embedded in a Hm-scale crustal volume. Heat transport by fault-borne fluid flow of tight gas sands in terms of Peclet number has been considered. They concluded that the crustal flow simulation using a single global numerical mesh may be the best.

In the paper "Modeling and Analysis of Magnetic Nanoparticles Injection in Water-Oil Two-Phase Flow in Porous Media Under Magnetic Field Effect," M. F. El-Amin et al. investigated the transport of magnetic nanoparticles in a water-oil, two-phase system under the influence of permanent magnetic field. They show that the location of the permanent magnet with respect to the inlet in a countercurrent two-phase system has significant effect in the flow field.

In the paper "Geofluid Systems of Koryaksky-Avachinsky Volcanoes (Kamchatka, Russia),” A. Kiryukhin et al. used seismic data to identify dyke swarms and potential heat sources to understand the role of volcanic eruptions in the adjacent thermal mineral springs. The isotope dating techniques showed the magmatic origins of $\mathrm{CO}_{2}$ and thermobiogenic origins of methane. A thermal hydrodynamic modelling was used to study the possible heat and mass sources to explain the observations of this interesting volcanic geofluid system.

In the paper "Gas-Water Flow Behavior in Water-Bearing Tight Gas Reservoirs," R. Cao et al. investigated the impact of mobile water on the gas flowing in tight pores. They presented mathematical models to describe flow behaviors of gas and water in tight gas formations, which account for the threshold pressure gradient, stress sensitivity, and relative permeability.

In the paper "Lattice Boltzmann Simulations of Fluid Flow in Continental Carbonate Reservoir Rocks and in Upscaled Rock Models Generated with Multiple-Point Geostatistics," J. Soete et al. used Palabos which is a software tool for particle-based modelling of classic computational fluid dynamics along with 3D pore network models to study fluid flow characteristics in continental carbonates. In addition, permeability simulations were performed on rock models generated with multiple-point geostatistics (MPS)

In the paper "Using BIB-SEM Imaging for Permeability Prediction in Heterogeneous Shales," C. J. A. Sinn et al. uses organic-rich shale samples from a lacustrine sedimentary sequence of the Newark Basin (New Jersey, USA). The study uses Broad Ion Beam polishing with Scanning Electron Microscopy (BIB-SEM) to obtain high-resolution pore scale images. This is used to determine pore system properties. The permeability is computed using the widely used CarmanKozeny models followed by validation through the experiments.

In the review paper "Flow and Transport in Tight and Shale Formations: A Review," A. Salama et al. presented a comprehensive review on the modeling of transport phenomena in tight and shale formations. They covered a wide range of phenomena occurring in shale and tight formations.

\section{Acknowledgments}

The guest editorial team would like to thank authors for all their contributions to this special issue. Given the space limitations, a number of high quality contributions could not be accommodated. The editors also wish to thank the anonymous reviewers for their time and dedication in providing 
their critical reviews that help focus the goal of this special issue. We hope that you will enjoy reading this special issue devoted to this exciting and fast-evolving field as much as we have done.

Amgad Salama

Shuyu Sun

Mohamed F. El Amin

Yi Wang

Kundan Kumar 

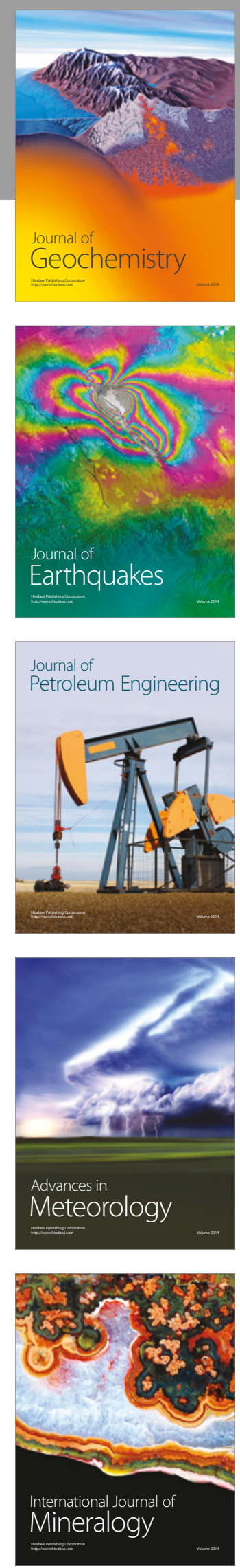
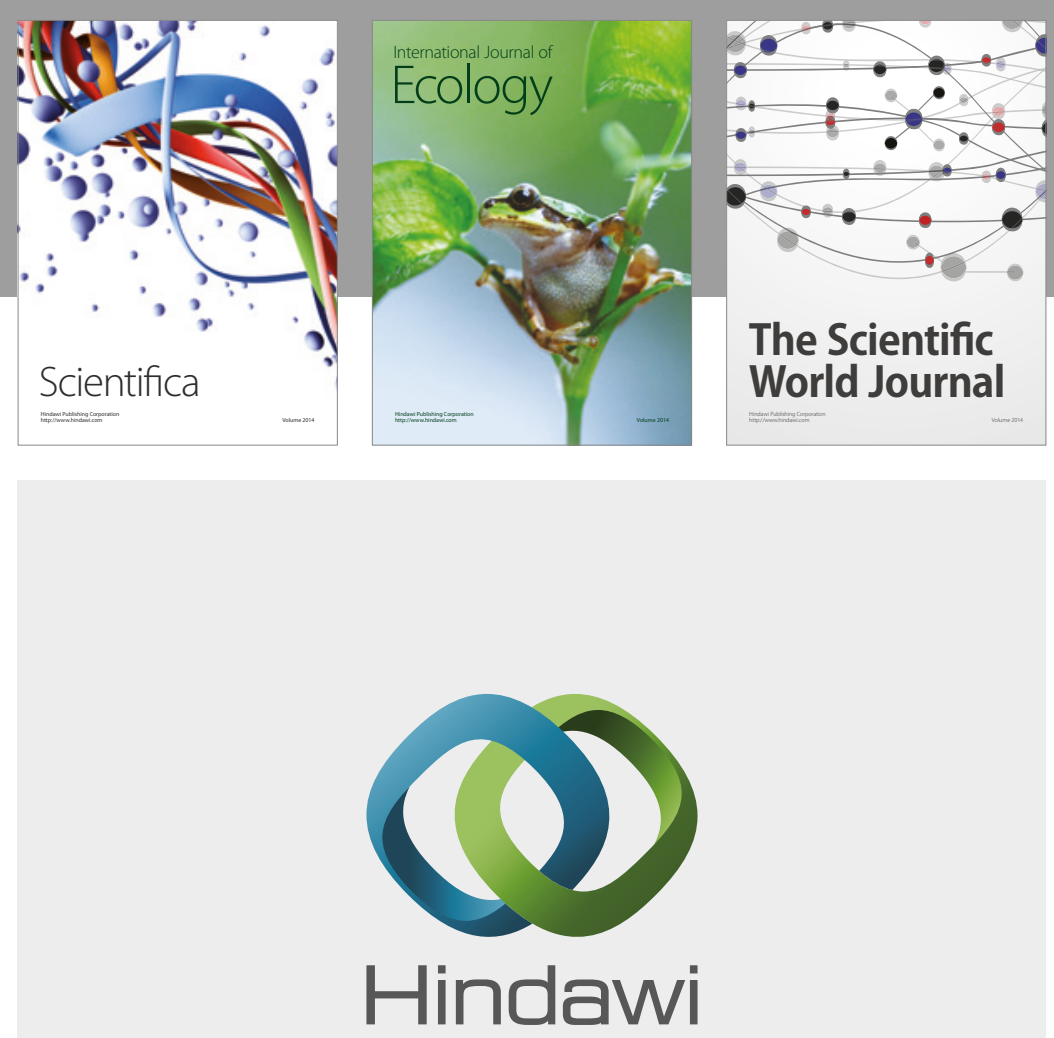

Submit your manuscripts at

https://www.hindawi.com
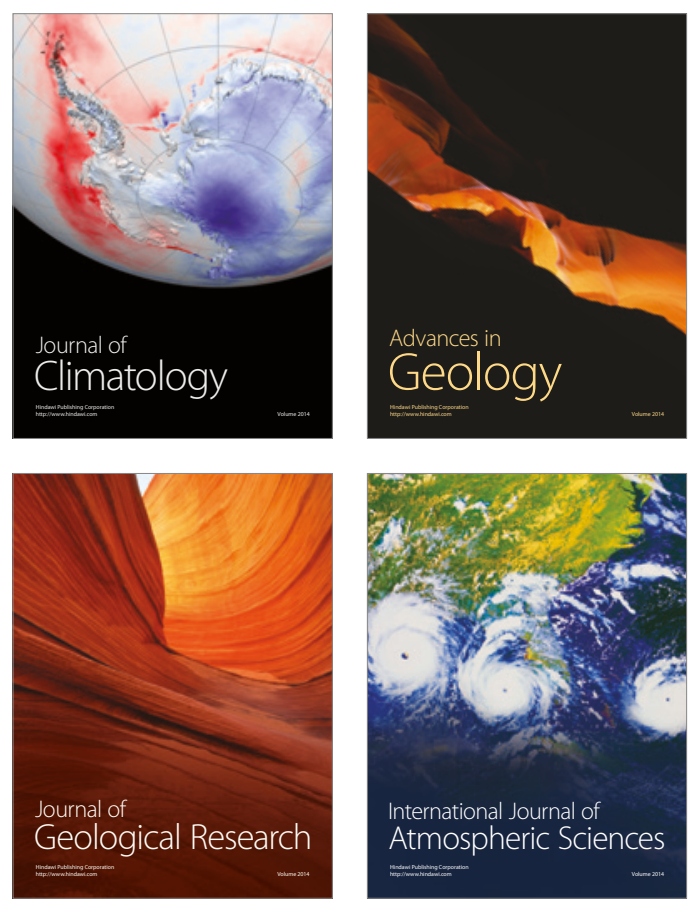

The Scientific

World Journal
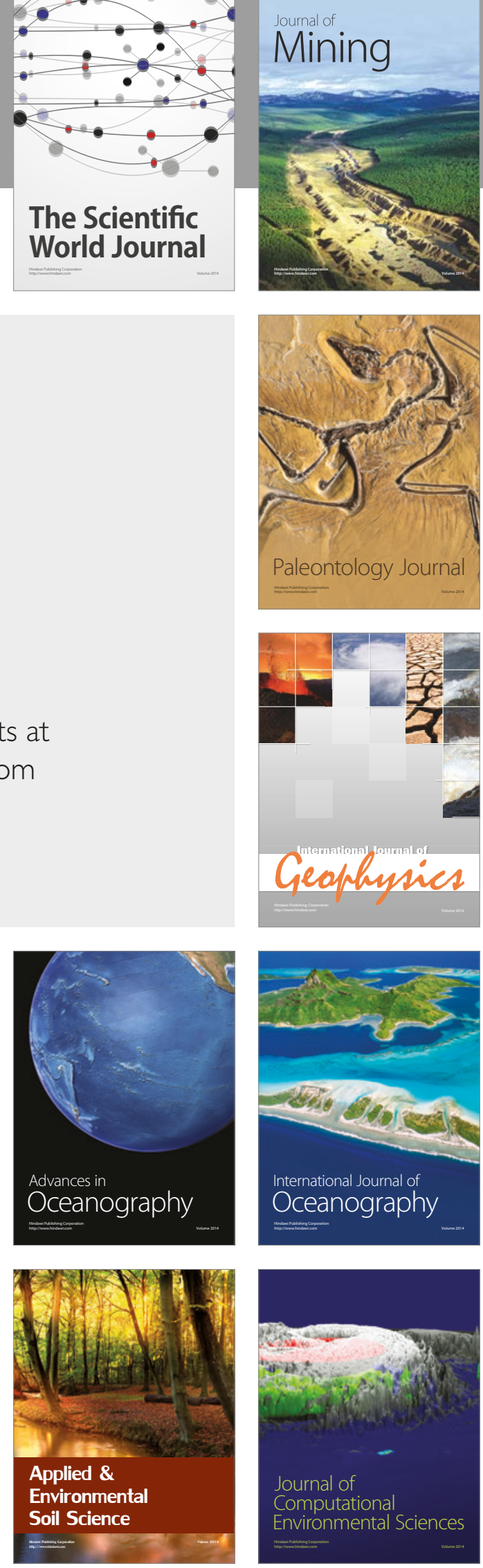